\title{
USO DE DROGAS POR ESTUDANTES DE MEDICINA DO CENTRO UNIVERSITÁRIO SÃO CAMILO
}

\author{
Gabriele Aparecida Camargo de Oliveira* \\ Gustavo Casanova Pinho* \\ Matheus Carvalho de Jesus* \\ Rodrigo Brasileiro de Souza* \\ Sabrina Santos Bifano* \\ Thiago Ribeiro Marcondes* \\ Yohran Cacciatori da Silva*
}

Sérgio Fernando Rodrigues Zanetta**

\section{Resumo}

O uso de drogas por estudantes de medicina é bastante comum, pois, pensando num contexto de pressão e pertencimento social, os alunos buscam através das substâncias uma forma de inserção social e de fuga de um cenário estressante. Devido a isso, o uso de substâncias deve ser alvo de estudo. O objetivo do estudo, portanto, é analisar o uso de drogas por estudantes de Medicina do Centro Universitário São Camilo, para levantar dados sobre a mudança do padrão de consumo, frequência e motivos para o uso. Para isso, foi feita uma pesquisa quantitativa por meio de um questionário de autopreenchimento via internet. Os resultados foram prevalência de uso de álcool, tabaco e maconha pelos estudantes, sendo o álcool a droga mais utilizada antes contexto universitário. Após entrar na faculdade, o lança-perfume e o metilenodioximetanfetamina (MDMA/ MD) ganham destaque nas substâncias mais utilizadas pela primeira vez na vida do estudante. A maioria dos estudantes afirma que a faculdade teve papel importante no aumento do consumo das substâncias. Deste modo, o tema o uso de drogas por estudantes de medicina deve ser estudado, para que seja possível compreender suas causas e pensar em estratégias de prevenção e de redução de danos.

Palavras - chave: Estudantes de medicina. Drogas. 


\title{
USE OF DRUGS BY MEDICAL STUDENTS OF CENTRO UNIVERSITÁRIO SÃO CAMILO
}

\begin{abstract}
The use of drugs by medical students is quite common, because, considering a context of pressure and social belonging, students seek through substances a form of social insertion and escape from a stressful scenario. Because of this, the use of substances should be a subject of study. The objective of the study, therefore, is to analyze the use of drugs by medical students from Centro Universitário São Camilo, to collect data on the change in consumption pattern, frequency and reasons of use. For this, a quantitative research was carried out through a self-completion questionnaire via internet. The results were prevalence of use of alcohol, tobacco and marijuana by students, with alcohol being the most commonly used drug before university context. Upon entering college, the 'lança- perfume' and MDMA / MD gain prominence in the most commonly used substances for the first time in a student's life. Most students says that college played a significant role in increasing substance use. Thus, the theme "The Use of Drugs by Medical Students" must be studied, so that it is possible to understand their causes and think of strategies of prevention and damage reduction.
\end{abstract}

Keywords: Medical students. Drugs. 


\section{INTRODUÇÃO}

Ao ingressar na universidade, o estudante de medicina é submetido a mudanças drásticas em sua carga horária e sua vida social, dessa maneira está exposto a um ambiente de estresse físico e emocional. Junto a isso, o maior acesso às drogas lícitas e ilícitas e a pressão de outros colegas para se inserir no grupo social faz com que o índice de consumo dessas substâncias entre os discentes de medicina seja maior que da população em geral. ${ }^{1,2,3}$.

Diversos estudos comprovam que dentre as drogas mais utilizadas estão: tabaco, maconha, álcool e tranquilizantes. ${ }^{2,3 .}$ Essas substâncias atuam em áreas do sistema nervoso central, relacionadas ao prazer e recompensa instantânea, sendo uma "válvula de escape" para o estresse e a cobrança diária que acomete o estudante de medicina. ${ }^{3 .}$

O uso abusivo dessas substâncias pode acarretar, além de prejuízos ao próprio indivíduo, problemas de saúde pública. O álcool é, por exemplo, a causa de inúmeras mortes por acidente de trânsito, aumento da violência interpessoal entre os jovens e doenças como cirrose e alguns tipos de câncer. Nas Américas, morrem cerca uma pessoa a cada dois minutos por causa do álcool. Estudos em 2003 comprovaram que 1400 estudantes universitários morrem anualmente devido ao uso abusivo de drogas. ${ }^{1,2,3,4}$.

Com base nas informações acima, fica evidente a importância desses estudos da prevalência do uso de drogas entre estudantes para que seja possível a elaboração de estratégias de redução de danos e conscientização sobre os riscos relacionados ao uso de substâncias lícitas e ilícitas. 


\section{METODOLOGIA}

Toda a coleta de dados foi realizada no mês de abril de 2019, através da aplicação de questionário de autopreenchimento formulado com base nos levantamentos bibliográficos e no questionário proposto pela Organização Mundial da Saúde. O objetivo dessa pesquisa quantitativa foi levantar informações sobre o uso de drogas na vida, nos últimos 3 meses, das seguintes substâncias: álcool, tabaco, maconha, cocaína, ecstasy, lança-perfume/ loló, metanfetamina, dietilamida do ácido lisérgico (LSD), MDMA/ MD. Além dessas informações, o questionário também apresentou questões sobre o uso antes e depois de ingressar na faculdade, sobre os motivos que levam ao uso e sobre a mudança no padrão de consumo desses estudantes nesse contexto.

Fizeram parte do projeto todos os alunos de medicina, do primeiro ao décimo segundo semestre, matriculados no Centro Universitário São Camilo.

A análise dos dados sobre o uso de drogas por estudantes de medicina do Centro Universitário São Camilo foi realizada através do total geral de respostas afirmativas colhidas, de acordo com o semestre do curso médico, com o sexo e com a idade.

Os questionários foram aplicados via internet. Juntamente ao questionário, o Termo de Consentimento e Esclarecimento foi apresentado para viabilizar a aquisição dos dados de forma legítima e isenta de não concordância por parte das pessoas que respondiam o questionário. Essa pesquisa é acompanhada de anonimato, sendo que a não concordância acarreta finalização automática do questionário. 


\section{RESULTADOS}

A partir da aplicação do questionário online, foram coletados os dados e organizados nas tabelas e gráficos a seguir. Dos entrevistados, apenas 1 não concordou com a pesquisa e, portanto, respondeu não concordo no Termo de Consentimento Livre e Esclarecido. Os outros 183, responderam ao questionário de forma concordante.

O perfil dos estudantes de Medicina, do Centro universitário São Camilo, que responderam ao questionário é em maior proporção feminino, entre 18 a 21 anos e pertencente ao ciclo básico do curso, dados esses apresentados na Tabela 1. Caracterizam-se, no geral, por pessoas jovens que não tiveram ainda papel atuante como médicos, mas que enfrentam grandes cargas teóricas.

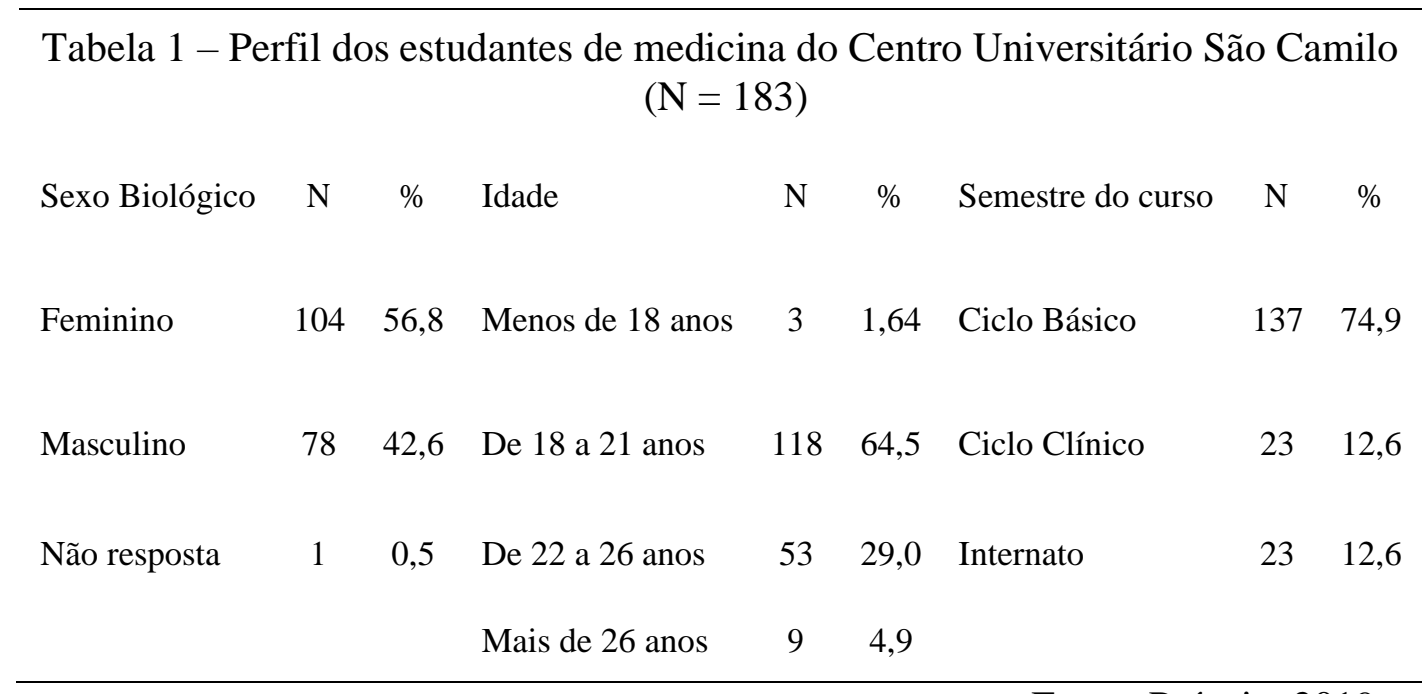

Fonte: Própria, 2019

No questionário, os entrevistados responderam quais das drogas citados já haviam utilizado pelo menos uma vez na vida. As drogas citadas foram: álcool, tabaco, maconha, cocaína, ecstasy, lança-perfume/ Lolo, metanfetamina, LSD, MDMA/ MD. Além disso, foi dada a opção de escolher que não havia utilizado nenhuma das drogas anteriormente citadas.

Dos resultados, temos uma prevalência do uso de álcool $(97,3 \%)$, maconha $(67,2 \%)$ e tabaco (59\%), respectivamente. O uso de Lança-perfume/ Lolo teve utilização significativa em relação às outras substâncias (50,3\%). Esses dados foram apresentados no gráfico 1. 
Gráfico 1 - Substâncias utilizadas pelos estudantes do Centro Universitário São Camilo

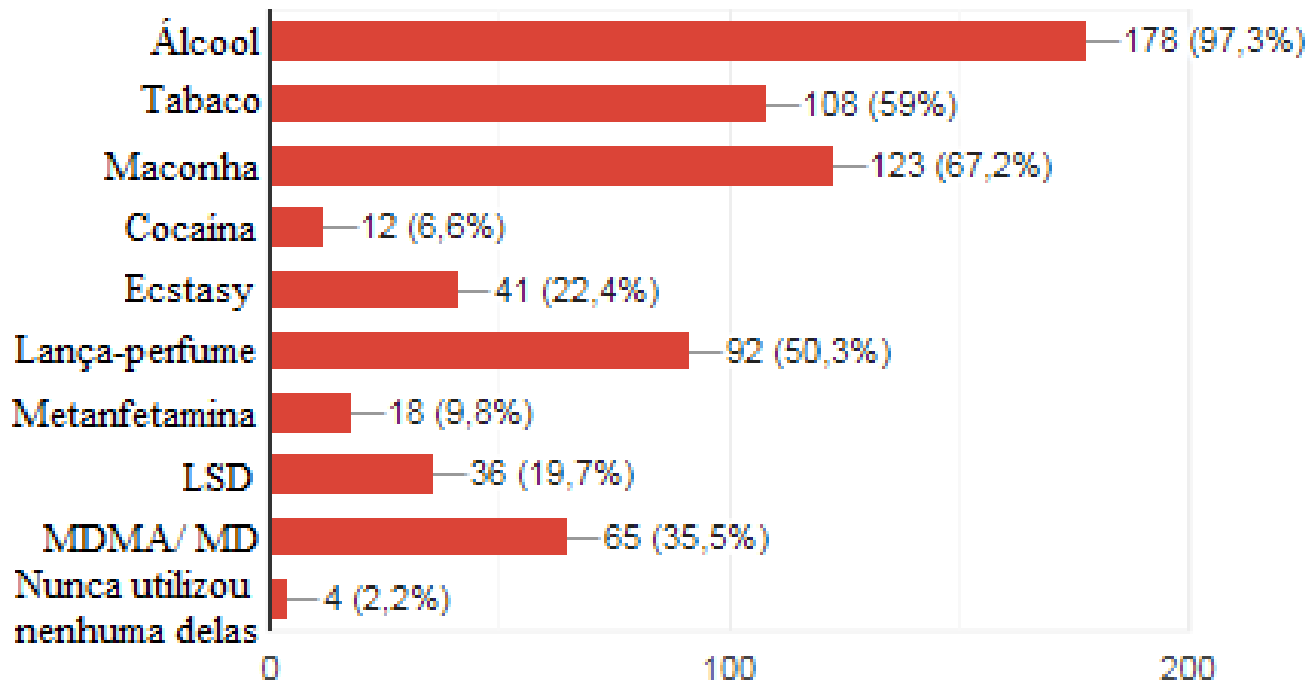

Fonte: Própria, 2019

Classificando o uso, foi questionado aos entrevistados quando eles começaram a utilizar as substâncias que mencionaram na questão anterior, se foram antes de ingressar na faculdade ou depois. O álcool foi a substância mais utilizada antes da faculdade (91,2\%). A cocaína, a metanfetamina, o ecstasy e o LSD são as substâncias mais relatadas como nunca utilizadas pelos entrevistados. Drogas como Lança-perfume/ Lolo e MDMA/MD tiveram início no uso, em maior proporção, com o ingresso na faculdade. Esses dados são apresentados no gráfico 2.

Gráfico 2 - Inicio do uso das substâncias pelos estudantes do Centro Universitário São Camilo

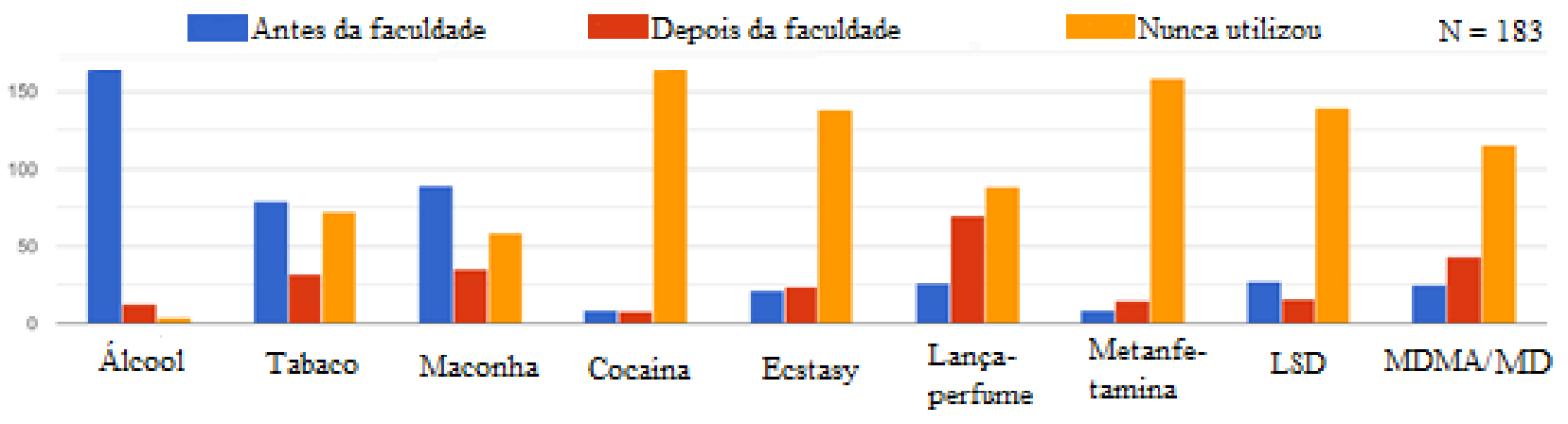

Fonte: Própria, 2019. 
Foram questionados aos entrevistados quanto a frequência do uso das substâncias anteriormente mencionadas nos últimos 3 meses. Esses dados são apresentados no gráfico 3.

A cocaína, o ecstasy, a metanfetamina e o LSD são as substâncias registradas como nunca utilizadas em maior proporção. O lança-perfume e o MDMA/MD competem com o álcool, com o tabaco e com a maconha, na frequência de 1 ou 2 vezes nos últimos 3 meses. O álcool é o mais utilizado semanalmente e mensalmente. A maconha e o tabaco são registrados como as mais utilizadas diariamente.

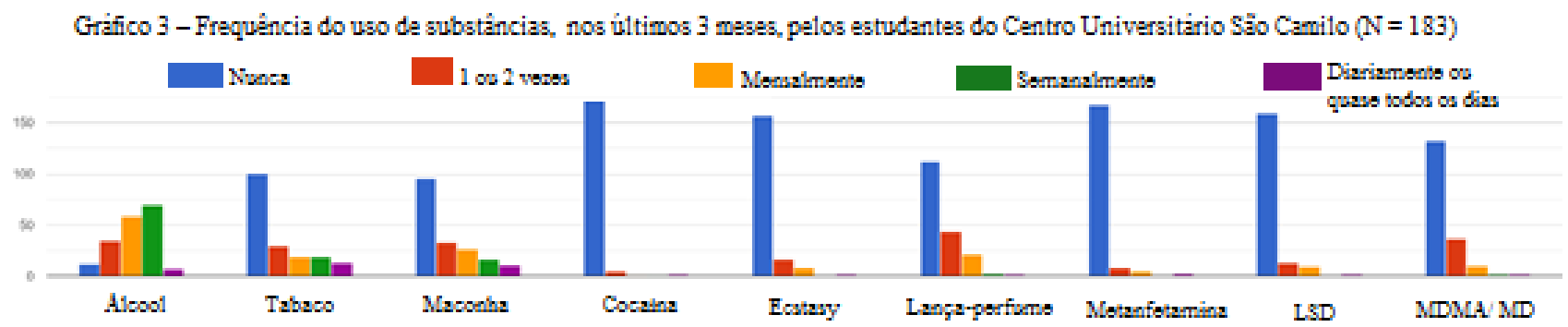

Fonte: Própria, 2019.

Além disso, foi levantado o motivo que levam aos estudantes ao uso das drogas anteriormente citadas. Os dados são apresentados na tabela 2.

Tabela 2 - Motivo do uso de drogas por estudantes de Medicina do Centro Universitário São Camilo $(\mathrm{N}=183)$

$\begin{array}{lcc} & \mathrm{N} & \% \\ \text { Recreativo } & 137 & 74,9 \\ \text { Influências de amizade } & 17 & 9,2 \\ \text { Desejo de fuga } & 12 & 6,6 \\ \text { Isolamento social } & 0 & 0,0 \\ \text { Hábito } & 3 & 1,6 \\ \text { Dificuldades na faculdade } & 3 & 1,6 \\ \text { Outros } & 11 & 6,0\end{array}$

Fonte: Própria, 2019. 
No questionário, foi perguntado também aos estudantes se houve um aumento no uso de drogas depois que ingressou na faculdade; $56,6 \%$ dos entrevistados afirmam que sim, enquanto 40,4\% dos entrevistados afirmam que não. Dos que responderam afirmativo, foram questionados quanto ao motivo que acreditam que tenham aumentado. Os dados são apresentados no gráfico 4.

\section{Gráfico 4 - Motivo pelo qual os estudantes de medicina do Centro Universitário São Camilo acreditam que o uso de drogas tenha aumentado depois do ingresso na faculdade $(\mathrm{N}=124)$}
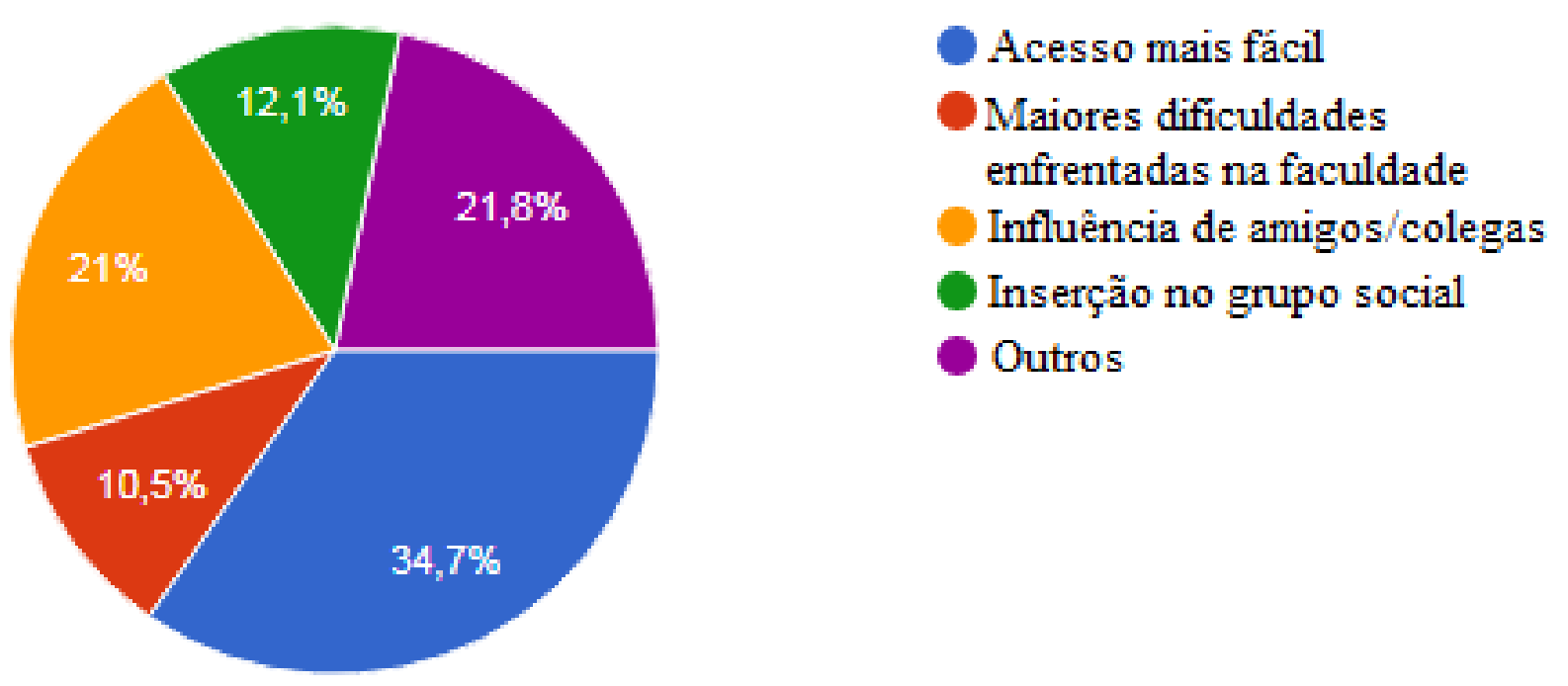

Fonte: Própria, 2019.

Além disso, foi questionado aos estudantes se eles acreditam que a faculdade teve responsabilidade pelo aumento do consumo de drogas pelos alunos; 56,3\% afirmam que sim, 18\% afirmam que não e $25,7 \%$ ficaram na dúvida quanto a responsabilidade do ingresso na faculdade.

No final do questionário foi deixado um espaço para que os estudantes pudessem compartilhar informações que acham pertinentes em relação ao tema. Um dos estudantes comenta que o seu uso diário é por motivos médicos, e o outro comenta que são utilizados para auxiliar os estudos, questões que não foram levadas em consideração quando se perguntou o motivo do uso.

Outro estudante relata a importância da legalização para um uso mais seguro, pois a ilegalidade não é um entrave para o uso. Além disso, eles acreditam que parte dos estudantes já tinha contato com as drogas e, que com o ambiente estressante, os estudantes procuram um refúgio nas drogas. As drogas teriam um acesso mais fácil na faculdade e seria uma forma de ascensão social. 
Abaixo está um dos comentários realizados pelos estudantes:

"O acesso a drogas, nos dias atuais, não é difícil e às vezes é facilitado pela condição econômica e social do indivíduo. Desse modo, a proibição não um entrave eficaz. Uma vez visada, a melhora da qualidade de vida da população (incluindo a dos estudantes de medicina), deve-se realizar um trabalho íntimo de diálogo e entendimento da realidade vivida por aquele indivíduo. Em alguns casos, o simples desabafo e a consciência de que há alguém preocupado com sua vida são suficientes medidas de conscientização (experiência própria)."

\section{DISCUSSÃO}

Para darmos início à nossa discussão, vale ressaltar que a maioria dos problemas sofridos por alunos de medicina já são previamente conhecidos. Em uma pesquisa feita na Venezuela, com 120 alunos em curso introdutório de medicina, consta que a grande maioria já espera passar por dificuldades psicológicas, emocionais, cognitivas e sociais dentro da faculdade; além disso, todos sabem da realidade das drogas nas universidades, e culpam a facilidade do acesso e também os problemas pessoais já citados. ${ }^{5}$

Dentre todas as drogas usadas pelos estudantes, o álcool é a que apresenta mais destaque em todas as pesquisas. É a droga mais usada e a com maior frequência de uso. Em nossa pesquisa, mais de $97 \%$ dos entrevistados já a utilizaram pelo menos uma vez na vida, 38\% consome mensalmente e quase $4 \%$ faz uso diário. Na maioria dos artigos utilizados, esse número não varia significantemente, e, até nos que apontam uma porcentagem menor de uso, o álcool segue campeão dentre as outras drogas. 
Em segundo lugar, nossa pesquisa aponta a maconha, em que mais de $67 \%$ dos estudantes já fizeram uso; e, logo em seguida, vem o tabaco, com 59\%. Apesar desses números, a quantidade de entrevistados que utiliza tabaco mensalmente e diariamente é maior em comparação dos que utilizam maconha. Um fato interessante é que na maioria dos artigos utilizados, o tabaco aparece em segundo lugar, gerando essa diferença em relação à nossa pesquisa.

Um diferencial para os nossos resultados é a quarta posição, que vem ocupada pelo lança perfume. Esse tipo de solvente nem é mencionado na maioria dos artigos ou aparece em porcentagens menores, enquanto na nossa pesquisa foi utilizado por mais de $50 \%$ dos entrevistados. Porém, a grande maioria só utilizou poucas vezes e apenas $2,7 \%$ faz uso semanal ou diário.

Vale, também, mencionar drogas como MDMA, Ecstasy e LSD, que correspondem, respectivamente, a 35,5\%, 22,4\% e 19,7\% de estudantes que já usaram. Os valores são relativamente altos, se comparados com outras pesquisas, mas, a grande maioria dessas pessoas que já utilizaram só fez uso apenas 1 ou 2 vezes durante os últimos três meses.

Apenas 2,2\% dos entrevistados nunca utilizaram nenhuma dessas drogas citadas e, outras drogas, como cocaína e metanfetamina, obtiveram porcentagens inferiores a 10\%, sendo, portanto, menos utilizadas não só em toda sociedade como, também, em nossa pesquisa.

A única droga que foi majoritariamente utilizada antes da faculdade foi o álcool, em que 92\% dos que utilizam começaram o uso previamente. Há outras drogas que também foram usadas pela maioria dos usuários antes, como o tabaco, a maconha e LSD, mas o número dos que começaram ou experimentaram depois de estarem na faculdade também é relevante.

Drogas como o Lança Perfume, MDMA e Ecstasy foram mais utilizadas após os estudantes estarem na faculdade. Além disso, 59,6\% dos entrevistados afirmaram que o uso de todas as drogas que utilizam foi aumentado ao entrarem na faculdade, e 56,3\% afirma que a faculdade é responsável por isso.

Esse aumento causado pela vida universitária foi justificado por $34,7 \%$ dos entrevistados por um acesso mais fácil, $21 \%$ diz que foi influência de amigos e também houve muitas respostas dizendo ser por conta de inserção em grupos sociais, dificuldades enfrentadas na vida acadêmica e outros motivos.

Ao pedir, no final da pesquisa, com as opiniões dos entrevistados sobre o tema, obtivemos repostas ressaltando a importância da mesma, pois o aumento do consumo de drogas é preocupante e não é abordado da forma certa. Também houve comentários citando o uso de drogas para fins medicinais e alguns argumentando positivamente sobre a legalização das drogas. 


\section{CONCLUSÃO}

Antes de apresentar uma conclusão, devemos levar em consideração as falhas que essa pesquisa apresenta. Um exemplo é o baixo número de pessoas que responderam, levando em consideração a quantidade de estudantes de medicina que a faculdade apresenta, além, claro, da possível não veracidade de algumas respostas, apesar do anonimato, pois, o medo da exposição pode levar a um filtro.

Mesmo com todas as possíveis falhas de amostragem, podemos concluir que o uso de drogas é realmente grande entre alunos de medicina do Centro Universitário apresentado. Essas drogas são utilizadas principalmente para uso recreativo e a frequência de uso, na maioria dos casos, aumentou após a entrada na faculdade.

Este estudo pesquisou alunos de medicina do Centro Universitário São Camilo e mostra, assim como outros estudos nacionais e internacionais, que há uma alta incidência de consumo de substâncias lícitas e ilícitas. O álcool é a droga mais prevalente com 97,3\% dos pesquisados, dos quais 90,7\% utilizaram antes de ingressar na faculdade. O uso semanal e mensal do álcool, nos últimos três meses, foi expressivo. O lança perfume teve incidência de 50,3\% e 75,0\% destes usaram depois da faculdade. Esses dados evidenciam a importância da elaboração de estratégias para prevenção e redução de danos do consumo de drogas na fase universitária. 


\section{REFERÊNCIAS}

1. GLORIA-GARCES, Carmen; VEDANA, Kelly Graziani Giacchero. Consumption of Central Nervous System Stimulants among Medical and Nursing Students at a Chilean University. SMAD, Rev. Eletrônica Saúde Mental Álcool Drog. (Ed. port.), Ribeirão Preto, v. $9, \quad$ n. 2, p. 64-69, ago. 2013. Disponível em: http://pepsic.bvsalud.org/scielo.php?script=sci_arttext\&pid=S1806$69762013000200003 \& \operatorname{lng}=p t \& n r m=i s o$. Acesso em: 31 maio 2019.

2. MONTOYA VASQUEZ, Erika Maria et al . Consumo percibido y uso de drogas lícitas e ilícitas en estudiantes universitarios en la Ciudad de Medellín, Colombia. Rev. LatinoAm. Enfermagem, Ribeirão Preto , v. 17, n. spe, p. 886-892, 2009 . Disponível em: http://www.scielo.br/scielo.php?script=sci_arttext\&pid=SO104$11692009000700020 \& \operatorname{lng}=e n \& n r m=i s o$. Acesso em: 31 maio 2019.

3. CANDIDO FJ1, SOUZA R, STUMPF MA, FERNANDES LG, VEIGA R, SANTIN M1, KLUTHCOVSKY A. The use of drugs and medical students: a literature review. Rev Assoc Med Bras (1992). 2018 Maio;64(5):462-468. Disponível em: https://www.ncbi.nlm.nih.gov/pubmed/30304147. Acesso em: 31 maio 2019.

4. RUIZ GONZALEZ, Mijail; CABALLERO ORTIZ, Valentín. Hábitos tóxicos en estudiantes de segundo año de medicina. Medisan, Santiago de Cuba , v. 17, n. 2, p. 230236 , feb. 2013. Disponível em: http://scielo.sld.cu/scielo.php?script=sci_arttext\&pid=S102930192013000200009\&lng=es\&nrm=iso. Acesso em: 31 maio 2019.

5. SAEZ, María Navarro de et al . Fatores de risco e propostas para a redução da demanda de drogas em estudantes de medicina de uma universidade venezuelana. SMAD, Rev. Eletrônica Saúde Mental Álcool Drog. (Ed. port.), Ribeirão Preto , v. 5, n. 2, p. 1-16, ago. 
$69762009000200002 \& \operatorname{lng}=p t \& n r m=i s o$. Acesso em: 31 maio 2019.

6. GARCIGA ORTEGA, Octavio; SURI TORRES, Carolina; RODRIGUEZ JORGE, Raúl. Consumo de drogas legales y estilo de vida en estudiantes de medicina. Rev Cubana Salud Pública, Ciudad de La Habana , v. 41, n. 1, marzo 2015 . Disponível em: http://scielo.sld.cu/scielo.php?script=sci_arttext\&pid=S0864$34662015000100002 \& \operatorname{lng}=e s \& n r m=i s o$. Acesso em: 31 maio 2019.

7. KERR-CORREA, Florence et al . Uso de álcool e drogas por estudantes de medicina da Unesp. Rev. Bras. Psiquiatr., São Paulo, v. 21, n. 2, p. 95-100, June 1999 . Disponível em: http://www.scielo.br/scielo.php?script=sci_arttext\&pid=S1516$44461999000200005 \& \operatorname{lng}=e n \& n r m=i s o$. Acesso em: 31 maio 2019.

8. OLIVEIRA JUNIOR, Hercilio Pereira de et al . Percepção dos estudantes universitários sobre o consumo de drogas entre seus pares no ABC Paulista, São Paulo, Brasil. Rev. Latino-Am. Enfermagem, Ribeirão Preto, v. 17, n. spe, p. 871-877, 2009 . Disponível em: http://www.scielo.br/scielo.php?script=sci_arttext\&pid=SO104$11692009000700018 \& \operatorname{lng}=$ pt\&nrm=iso. Acesso em: 31 maio 2019.

9. HENRIQUEZ, Patricia Cid; CARVALHO, Ana Maria Pimenta de. Perceptions of drugs benefits and barriers to quit by undergraduate health students. Rev. Latino-Am.

Enfermagem, Ribeirão Preto , v. 16, n. spe, p. 621-626, Aug. 2008 . Disponível em: http://www.scielo.br/scielo.php?script=sci_arttext\&pid=S0104-

$11692008000700019 \& \operatorname{lng}=e n \& n r m=i s o$. Acesso em: 31 maio 2019.

10. KOWALCZUK K, KRAJEWSKA-KUŁAK E. Exposure to Psychoactive Compounds amongst Students of Medical University. 10 Cent Eur J Public Health. 2017 Sep;25(3):200205. Disponível em: https://www.ncbi.nlm.nih.gov/pubmed/29022678. Acesso em: 31 maio 2019. 
11. AYALA EE, ROSEMAN D, WINSEMAN JS, MASON HRC. Prevalence, perceptions, and consequences of substance use in medical students. Med Educ Online. 2017;22(1):1392824. Disponível em: https://www.ncbi.nlm.nih.gov/pubmed/29072119. Acesso em: 31 maio 2019. 

